\title{
Lidil
}

Revue de linguistique et de didactique des langues

$48 \mid 2013$

L'émotion et l'apprentissage des langues

\section{Activités de production orale et émotions : gérer la complexité dans l'instant de l'échange}

Emmanuelle Maitre de Pembroke

\section{OpenEdition}

\section{Journals}

Édition électronique

URL : http://journals.openedition.org/lidil/3325

DOI : 10.4000/lidil.3325

ISSN : 1960-6052

\section{Éditeur}

UGA Éditions/Université Grenoble Alpes

\section{Édition imprimée}

Date de publication : 1 novembre 2013

Pagination : 157-169

ISBN : $978-2-84310-260-8$

ISSN : 1146-6480

\section{Référence électronique}

Emmanuelle Maitre de Pembroke, «Activités de production orale et émotions : gérer la complexité dans l'instant de l'échange », Lidil [En ligne], 48 | 2013, mis en ligne le 01 mai 2015, consulté le 30 avril 2019. URL : http://journals.openedition.org/lidil/3325; DOI : 10.4000/lidil.3325 


\title{
Activités de production orale et émotions : gérer la complexité dans l'instant de l'échange
}

\author{
Emmanuelle Maitre de Pembroke*
}

\begin{abstract}
RÉSUMÉ
Les personnes expatriées à Paris vivent une situation d'exolinguisme parfois éprouvante. Les difficultés pour entrer dans les échanges portent sur de nombreux niveaux d'ordre linguistique, paralinguistique, métacognitif et pragmatique. Cet article présente un travail de recherche qui vise à explorer les situations de difficulté de communication en situation exolingue. Par le biais d'entretiens qualitatifs, l'analyse de moments précis d'interactions permet de dégager les émotions ressenties, le contexte de leur émergence et leurs causes. Ce travail met en relief l'importance de la compréhension des éléments contextuels et le rôle de l'incorporation des grilles sémiologiques.
\end{abstract}

\section{ABSTRACT}

The expatriated people arriving in Paris have difficulties to communicate in the daily life. Numerous levels are concerned: linguistic, paralinguistic, metacognitive and pragmatic. This article presents a research on difficulties of communication. Thanks to qualitative interviews, we analyze precise moments of highly emotional interactions. We study the context and the causes of emotions. This work underlines the importance of contextual informations in communication and the role of embodied knowledge to understand.

Les personnes expatriées souhaitant s'intégrer dans la vie quotidienne française vivent une situation d'exolinguisme parfois éprouvante. Les difficultés pour entrer dans les échanges portent sur de nombreux niveaux d'ordre linguistique, paralinguistique, métacognitif, pragmatique : gérer la charge cognitive, comprendre les indices paraverbaux,

* Maitre de conférences, Laboratoire REV-CIRCEFT, Université Paris Est Créteil. 
saisir les éléments du contexte interlocutif, savoir co-construire son image identitaire et celle de l'autre. Lorsque la coopération est réussie, les deux interlocuteurs visent à réduire les écarts dans un processus de compréhension mutuelle. Les situations marquées par des émotions fortes émergent lorsque cet ajustement échoue. Entrent en jeu tout à la fois une prise de conscience d'un déficit linguistique et le sentiment que leur grille de lecture des indices contextuels est défaillante. Mené auprès de communautés expatriées, le travail présenté explore les situations de difficulté de communication. Des entretiens qualitatifs contextualisés visent à documenter et analyser le vécu expérientiel de situations d'échange marquées par les émotions. La centration sur des moments précis permet de dégager les émotions ressenties, le contexte de leur émergence et leurs causes. Les travaux de Damasio (2010) considèrent les émotions comme une évaluation de l'environnement, passant par le ressenti corporel qui prépare le corps à réagir de façon adaptative. Cependant, ce processus est inconscient et non réflexif. Il est influencé par des facteurs culturels, sociaux et contextuels. Les entretiens font ressortir l'importance de la saisie des éléments contextuels et pragmatiques dans la co-construction de la compréhension et le fait que ces connaissances, incorporées par les natifs, sont difficiles à intégrer par les personnes qui ne disposent pas des grilles sémiologiques de la communauté d'accueil.

\section{Cadre théorique}

\subsection{L'oral : une complexité de procédures à gérer}

Les travaux de psycholinguistique mettent en avant la complexité des processus cognitifs mobilisés lors des échanges oraux. Du fait de leur simultanéité, la charge attentionnelle est élevée (Gaonac'h, 1987). Les compétences requises pour comprendre un discours lors d'une interaction relèvent de nombreux niveaux : sociolinguistiques sur la situation de communication, socio-psychologiques sur le producteur du message et les circonstances de l'échange, discursives sur le type de discours concerné, linguistiques sur le code utilisé, référentiels sur la thématique invoquée, culturels sur la communauté à laquelle appartient le producteur du message (Gremmo \& Holec, 1990). Le modèle interactif de la compréhension met l'accent sur les compétences de saisie dans l'environnement (contenus linguistiques et indices), en lien avec la mobilisation de connaissances antérieurement intégrées sur chacun 
des niveaux (Fayol, 1997). Cette interaction entre connaissances et contexte est aussi mise en avant dans les travaux sur la production orale. Selon Levelt (1989), une boucle de production permet au locuteur de réguler son discours en temps réel pour l'ajuster au contenu de sa pensée en fonction des éléments contextuels. La difficulté pour entrer dans l'échange est liée à deux éléments fondamentaux. Le premier est donc la complexité. Au traitement linguistique s'ajoute la mobilisation de connaissances pragmatiques portant sur la relation et son contexte. D'autre part, ces processus de perception et traitement du sens ne sont pas conscients. Ils sont automatisés et nécessitent une activité de prise de conscience pour être optimisés. Les processus métacognitifs qu'il faut mobiliser pour prendre conscience de son discours dépassent les processus métalinguistiques, dans la mesure où ils portent tout à la fois sur les ressources linguistiques à disposition, l'évaluation du contenu de sa propre pensée et les paramètres relationnels et contextuels (Trévise, 1996). Les informations non verbales (mimiques, gestuelle) et paralinguistiques (prosodie, courbe mélodique) sont particulièrement marquées par des mécanismes automatisés, c'est-à-dire non conscients (Mondillon \& Tcherkassof, 2009). Audibert (2005), Banziger et Scherer (2005) soulignent le rôle prédominant de la prosodie dans l'interprétation immédiate du contenu intentionnel et émotionnel du message reçu. La difficulté d'une intercompréhension réside dans le fait que ces données paraverbales font surgir des émotions fortes exprimées par un ressenti non conscientisé. Ces travaux ont l'intérêt, non seulement de dégager les composantes prosodiques en jeu, mais aussi de recenser les émotions afférentes.

\subsection{Les situations de communication exolingue}

Les personnes rencontrées lors des entretiens sont des personnes expatriées en France qui doivent communiquer en situation exolingue. Par opposition à la communication endolingue qui concerne des locuteurs utilisant une langue maternelle commune, la communication exolingue rassemble des locuteurs dont la langue maternelle diffère (Porquier, 1984). La situation exolingue est fragilisante pour les locuteurs qui ne possèdent pas les outils de communication en usage dans le contexte d'accueil. Impliquant des inégalités ou des asymétries, elle limite la possibilité d'expression de son identité. En particulier, la situation exolingue est caractérisée par un déséquilibre dans les savoirs linguistiques mobilisés par les interlocuteurs, ce qui est facteur de stress et de vulnérabilité dans l'échange. Pour pallier ces déficits linguistiques, les 
locuteurs mobilisent certaines stratégies permettant de les compenser par la saisie des «indices de contextualisation» (Gumperz, 1989). Ces indices portent particulièrement sur l'interprétation (souvent non consciente) d'informations paralinguistiques et kinesthésiques (Picard, 1992). Les auteurs soulignent que cette situation engageant des locuteurs natifs et non natifs de la langue nécessite une coopération et une co-construction de l'interaction. En effet, une situation d'échange exolingue, même faible sur le plan linguistique, peut être réussie si les locuteurs sont investis dans un contrat de communication et saisissent tous les éléments contextuels et paralinguistiques favorisant la compréhension mutuelle. L'approche interactionniste met l'accent sur les stratégies partagées de compensation et d'ajustement visant à réduire les écarts (Alber \& Py, 1985).

\subsection{Approche communicative et contextualisée de l'échange}

La difficulté de saisie et de compréhension des paramètres contextuels de l'échange est cependant extrêmement difficile pour une personne non native de la culture d'accueil. Les travaux sur le contexte interlocutif (Auzanneau, 2007) soulignent la difficulté d'interpréter les données situationnelles de l'échange. Les travaux anthropologiques de la cognition située soulignent l'importance du milieu d'origine et du contexte dans l'incorporation des grilles d'interprétation. Le terme d'«incorporation» souligne comment l'individu intègre ses perceptions et modes de communication à travers son environnement (Varela, 1993 ; Damasio, 2010). Bien intégrées, ces grilles de lecture ne sont pas conscientisées. Ce n'est que lors d'échecs de la relation que la personne prend conscience de modes communicationnels différents des siens. Ces échecs sont alors vécus comme des blessures identitaires. Dans un contrat communicatif dont les implicites sont partagés, l'ensemble des échanges entrent dans des rituels qui visent à «préserver la face» (Goffman, 1967). Pour cet auteur, la méconnaissance du contexte entraine des situations de perte de la face extrêmement fortes émotionnellement. C'est pourquoi l'échange est un risque important qui met en jeu une image de soi et une image de l'autre. Les travaux sur la pragmatique montrent bien l'impact du message sur la relation qui lie les interlocuteurs (dimension perlocutoire). En particulier les actes de langage, tels que les remerciements par exemple, ont un pouvoir bien plus fort que le seul contenu linguistique des mots utilisés (Searle, 1972; Austin, 2005). 


\section{Méthodologie}

En situation d'expatriation, les personnes vivent une situation exolingue dans laquelle les difficultés communicationnelles sont plus ou moins fortes. Le déséquilibre dans les capacités d'expression face aux interlocuteurs natifs peut provoquer des émotions marquées, d'autant plus que les personnes expatriées sont en quête d'intégration. Un autre déséquilibre entre en jeu évoqué par Porquier (1984) lorsqu'il parle de «micro-contextes» (p. 239) : celui dû aux exigences de positionnement hiérarchique lié à l'entreprise. Ainsi, à des difficultés d'insertion, s'ajoutent des difficultés d'expression de l'identité professionnelle.

Cet article présente l'analyse d'entretiens menés auprès de vingtquatre adultes en situation d'expatriation. Ceux-ci doivent acquérir la langue française en immersion dans un contexte culturel différent du leur. Ces personnes travaillant au sein de grandes entreprises multinationales (IBM, General Electric) viennent en France pour une durée de trois à six ans. Elles bénéficient de cours de français langue étrangère au sein de l'entreprise et c'est dans ce cadre que les entretiens ont été menés. Nous avons affaire à un panel large d'origine géographique puisque les cinq continents sont représentés dans l'échantillon. Ce ne sont pas tant les cultures d'origine qui nous intéressent - puisque ces personnes sont riches de multiples subcultures acquises lors de leur parcours internationaux (Porcher \& Abdallah Pretceille, 1998) - mais les moments marquants de découverte de nouveaux fonctionnements interactionnels empreints de contenus émotionnels. Ce sont donc les moments de communication vécus comme difficiles ou forts qui sont explorés.

Les travaux présentés ici prennent appui sur des entretiens qualitatifs individuels. La méthodologie utilisée pour mener ces entretiens s'appuie sur la démarche de l'entretien d'explicitation qui permet de «reconscientiser» une situation vécue (Vermersch, 2008). Ce type d'entretien a pour but la prise de conscience de mécanismes pré-réfléchis (Piaget, 1974), inscrits dans la mémoire autobiographique, qui n'apparaissent pas d'emblée à la conscience. Cet entretien permet un retour réflexif sur le vécu d'un moment précis. Sa vocation première est la description avec une granularité fine de tous les aspects d'une situation singulière. Il permet à la personne de reprendre contact avec des comportements verbaux et non verbaux expérimentés. Les questions posées portent sur le contexte de l'échange évoqué, puis sur les microfonctionnements interactionnels : ce qui a été échangé, sous quelles 
formes, associé à quel ressenti. Reviennent alors à la mémoire les prises d'indices, les choix et les ressentis lors d'un moment contextualisé du passé.

L'objectif des entretiens était de retrouver des moments d'interaction au cours desquels la prise de parole avait été marquée par des émotions et de dégager les composantes constitutives de ces échanges. Ce type de questionnement amenant à des ressentis forts et parfois difficiles, un contrat de communication particulièrement clair était mis en place. Il visait à respecter ce que la personne souhaitait ou non explorer. D'autre part, les vécus chargés émotionnellement étaient revisités sous leurs aspects positifs. En effet, étaient dégagés les savoir-faire communicationnels appris et les stratégies développées pour favoriser l'échange. Ma situation d'interviewer était celle d'une recherche impliquée telle qu'elle est définie par l'anthropologie. En effet, ces entretiens ont été possibles parce que j'étais la personne chargée d'accompagner leur intégration et de nombreux moments d'écoute avaient déjà eu lieu. Les relations qui m'unissaient à eux étaient établies sur une durée longue faite de confiance mutuelle et de découvertes partagées.

Le corpus est constitué de la transcription des vingt-quatre entretiens menés sur une soixantaine de minutes chacun. Il est analysé de manière thématique à partir des thèmes se dégageant de façon récurrente (Miles \& Huberman, 2003).

\section{Résultats}

L'analyse de ce corpus permet de distinguer différents niveaux de difficultés. Certaines relèvent de la charge due au traitement cognitif de la parole. D'autres relèvent de difficultés à interpréter correctement le contenu émotionnel véhiculé par des dimensions paraverbales. Enfin, le positionnement identitaire permettant d'adopter les formes d'expression correctes envers autrui est difficile. Les obstacles qui touchent à l'identité provoquent des émotions profondes. Des difficultés particulières ont été relevées dans des actes de langage tels que le remerciement, la négation et l'argumentation.

\subsection{Difficultés dues au traitement linguistique}

De l'ensemble des entretiens ressort la difficulté à traiter de façon simultanée la construction du sens des propos et la correction linguistique. Conformément aux travaux de psycholinguistique cités, la charge 
cognitive est forte. Selon les individus, l'attention est portée sur un des niveaux : phonétique, lexical, syntaxique, pragmatique. Par exemple, de nombreux ressortissants de langue japonaise ou chinoise expriment la difficulté de se concentrer sur les aspects articulatoires et de conserver le contenu de sens, ainsi que le fil de leur pensée (cohérence globale). Pour beaucoup des personnes interrogées, la recherche du mot correct en mémoire lexicale provoque la perte des autres niveaux. La surcharge cognitive requise pour ajuster tous les niveaux de la prise de parole provoque l'émergence d'émotions liées au découragement. Dans la mesure où la pensée ne trouve plus la fluidité et le mode d'expression auxquels elle est habituée dans la langue d'origine, apparait aussi le terme de «perte d'identité». «Comme je parle lentement et sans vocabulaire, on ne prend pas au sérieux ce que je dis.» Les personnes expriment ainsi un lien fort entre capacité d'expression et positionnement identitaire dans la relation à l'autre. «Je ne sais pas trouver les bons mots pour me présenter et surtout, pour dire qui je suis.» Surgit également le sentiment de régression dans la mesure où le bagage lexical de la langue étrangère en cours d'apprentissage correspond à celui d'un enfant. Un sentiment de perte d'estime et de capacité d'expression de son identité sociale provoque frustration et colère. «Je ne m'exprime pas avec le niveau que je souhaiterais. Ce que je dis ne correspond pas à ma pensée et cela me met très en colère.»

Ces difficultés de traitement simultané des procédures cognitives mettent en relief différentes dimensions temporelles. Tout d'abord, les personnes ne parviennent pas à gérer en parallèle la complexité des procédures : phonologiques, lexicales, syntaxiques, textuelles et pragmatiques. En cours de FLE, elles se sentent rassurées lorsqu'elles peuvent centrer leur attention tantôt sur un niveau, tantôt sur un autre, dans des activités d'entrainement, dans le but d'automatiser des modes de communication socialement corrects. Par exemple, s'entrainer à une courbe mélodique appropriée pour saluer ou remercier. Il leur semble que maitriser un bon «démarrage de la relation» leur permettrait ensuite de s'exprimer avec confiance, même si le débit reste lent et hésitant. Autre dimension temporelle chargée émotionnellement : celle de l'alternance des prises de parole. En effet, en langue étrangère, la charge attentionnelle est si forte que les personnes ont besoin de plus de temps pour prendre la parole. Or, il leur semble ne jamais pouvoir saisir leur tour au bon moment. «Je veux parler mais je ne peux jamais placer ma phrase.» «Tout le monde parle en même temps. Les autres sont plus rapides que moi alors je ne peux intervenir au bon moment.» Selon 
le point de vue des personnes interrogées, les échanges verbaux français sont très rapides et les discours se chevauchent. Ces remarques confirment les travaux précédents sur la communication interculturelle (de Pembroke, 1998) qui ont amené à introduire les concepts de «mode d'échange polyphonique» : chevauchement et enchâssement des discours et «mode d'échange monophonique» : ralentissement et séquentialisation des discours. Recourir à un mode monophonique (chacun son tour) et laisser un temps de concentration au locuteur semble un facteur réconfortant. Certains entretiens soulignent que ce temps n'est pas donné dans les cours de FLE suivis en entreprise, car les temps de silence ne sont pas acceptés. L'enseignant passe très vite d'un locuteur à un autre. «Il n'y a jamais de silence pour pouvoir se préparer à parler. Alors pour moi, c'est impossible.» Des stratégies différentes apparaissent dans la gestion des frustrations, lesquelles relèvent de différences culturelles. Les ressortissants de cultures latines sont à l'aise avec la gestion polyphonique et la rapidité des échanges, car elles se lancent dans la parole même si leur niveau de correction linguistique n'est pas parfait. «J'aime parler. Même si mon niveau n'est pas correct, je m'en sors toujours pour dire ce que je veux.» Les personnes de cultures asiatiques, marquées par la lenteur et la monophonie, restent muettes. La frustration est compensée par la valeur qu'elles accordent au silence et à l'écoute. «Pour nous, le silence est précieux. Alors, si certains sont fâchés de ne pas pouvoir parler, pour moi, ce n'est pas grave : je progresse en écoutant.»

\subsection{Difficultés liées à la courbe mélodique}

En conformité avec les travaux évoqués, les éléments prosodiques sont ressortis très clairement dans les difficultés à interpréter le contenu émotionnel d'un message en français. Cela provoque en retour des réactions émotionnelles fortes que les personnes apprennent à gérer au fur et à mesure que leur temps de séjour en France s'allonge. Les personnes remarquent une courbe mélodique particulièrement accentuée lors des actes de politesse : salutations, remerciements. La voix prend alors une tonalité chantante et la hauteur change pour se situer dans des tons plus élevés. Les femmes perçoivent bien ces dimensions illocutoires contenues dans le message et adoptent volontiers les courbes mélodiques qu'elles assimilent à de la bienveillance. En revanche, beaucoup d'hommes adoptent difficilement ces tonalités qu'ils jugent trop féminines. Acquérir ces modulations de voix entre en contradiction avec l'image de leur identité. La plus grosse difficulté réside dans l'interpré- 
tation exacte des intentions de communication portées par la courbe mélodique. De nombreux malentendus et conflits ont été relatés. Éclats de voix, intonations soudainement accentuées : les témoignages évoquent l'émotion forte ressentie face à une courbe mélodique qu'ils ne comprenaient pas. «J'ai eu l'impression que mon patron était en colère et j'ai paniqué. J'ai compris après qu'il était juste enthousiaste.» «Pour les Japonais, le ton de voix des Français est perçu comme agressif et surtout trop émotionnel. Cela provoque chez nous un certain mépris pour la personne qui ne sait pas contrôler ses émotions et s'exprimer posément. Surtout, nous avons la sensation d'être agressés.» Même si le contenu du message n'est pas entièrement décrypté, une force perlocutoire se dégage et l'intention de communication est immédiatement traitée selon une grille de lecture inconsciente relevant de la culture d'origine. Les personnes évoquent d'une part une difficulté à prendre conscience de ces paramètres non explicités. D'autre part, même si la personne conçoit ces décalages d'interprétation, il lui est difficile de se distancier du contenu émotionnel. L'interprétation de la courbe mélodique semble être de l'ordre de l'immédiateté et les émotions surgissent dans le même temps : peur, colère. Les personnes parlent de réactions «épidermiques», comme si «le message était interprété par mon corps et non pas par ma tête». "C'est mon corps qui réagit, qui me dit que ça ne va pas.» Ces témoignages montrent bien comment s'opère de façon inconsciente l'incorporation des composantes relationnelles. La récurrence de ces remarques souligne l'importance de la prosodie dans l'impact émotionnel. Il est donc nécessaire d'apprendre à se distancier, en analysant les variations phonétiques d'ordre culturel. En cours de langue, les activités sur ces variables mélodiques sont fondamentales. Au fur et à mesure des échanges, les personnes ont pu prendre conscience que seul le locuteur était en mesure d'expliciter l'intention contenue dans la courbe mélodique. En d'autres termes, au cours de leur séjour en France, certains ont appris à se distancier de leur grille d'interprétation immédiate et à s'appuyer sur d'autres prises d'indices (non verbaux, par exemple). «Au début, j'étais bouleversée par les tons de voix. Après trois années en France, je sais qu'il n'y a pas d'intention négative quand le volume est fort ou quand l'intonation est marquée.» Ces prises de conscience leur ont permis aussi de moduler leur propre courbe mélodique en fonction du destinataire.3.3. Difficultés liées aux positionnements

Dans le cadre des échanges oraux, les marqueurs de positionnement identitaire jouent fortement sur le ressenti émotionnel. La conviction 
d'avoir ou non droit à la parole va marquer l'implication émotionnelle. Les marqueurs sociologiques sont pertinents pour analyser les positionnements des interlocuteurs : âge, sexe, statut. Ainsi, les entretiens montrent que dans certaines cultures, les femmes ne peuvent prendre la parole à la suite d'un homme. De même, une personne plus jeune ne peut donner son opinion face à une personne plus âgée. En cours de FLE, l'asymétrie de l'âge est renforcée par le statut du professeur. «Une fois, j'ai levé la main et le prof ne m'a pas interrogée. Je ne lève plus la main car je crois que je n'ai pas le droit.» Dans certaines cultures, l'enseignant est dans une position si haute que l'échange verbal est difficile ou est réglé selon des règles conventionnelles fortes. «Pour moi, le professeur est tout en haut et je suis tout en bas. Ce n'est pas correct de prendre la parole.» De nombreux témoignages évoquent ces difficultés de prise de parole. Ces croyances font surgir des émotions fortes lorsqu'il faut se lancer dans l'échange : perte de repères, stress, tremblements, accélération de la respiration. D'autre part, plus les modalités de prise de parole diffèrent des représentations que l'on se fait, plus les émotions sont difficiles à gérer dans le même temps que la parole. «Je suis une femme et si je dois m'exprimer devant un homme, je m'inquiète trop.» D'où ce qui est évoqué : le blanc total, la perte du contenu. Pour certaines personnes, l'anticipation de ces émotions les amène d'emblée à renoncer à toute intervention.

Ces marqueurs de positionnement identitaire sont corrélés avec des valeurs différentes attribuées à la parole et au silence. Dans les cultures où le silence est fortement valorisé, faire le choix de se taire est cohérent avec le système de valeurs selon lequel «le silence est une vertu». En revanche, pour d'autres personnes, ne pas réussir à identifier les marqueurs et donc ne pas pouvoir se positionner correctement pour prendre la parole est très frustrant. Des différences fortes marquent aussi la valeur accordée au langage par rapport à l'action. Agacement, impatience, désintérêt accompagnent les temps d'échange trop longs pour les personnes qui se disent d'une culture de l'action (évoquée par les Anglo-Saxons). Tout au contraire, dans certaines cultures, l'expression orale est conçue comme une action importante de lien avec autrui et la fonction phatique (établissement de la relation) joue un rôle prédominant; «Prendre le temps de parler est valorisé».

\subsection{Difficultés liées à certains actes de parole}

Dans l'ensemble des entretiens, certains actes de parole ont été relevés comme fortement marqués par l'émotion. Remercier, par exemple, ne 
consiste pas seulement à énoncer une formule toute faite. En effet, dans certaines langues, le remerciement est conçu comme condescendant. «Si je remercie, je mets la personne au niveau du sol.» Choc émotionnel fort pour les personnes qui, en référence à leur système, n'usent pas de ce procédé et se voient qualifiées d'impolies. Difficultés également pour trouver le bon vecteur de sa gratitude lorsque sa culture ne la manifeste que par du langage gestuel. «Quand j'exprime la gratitude avec mon visage, comme nous le faisons dans mon pays, les personnes françaises ne le voient pas.» Plus impliquant encore est le fait de nier. Dans certains systèmes linguistiques, des procédés permettent d'éviter l'expression verbale de cette négation : soit par des phrases détournées, soit par un ton de voix très bas («un oui très peu prononcé»), soit par une gestuelle fine («une expression de visage très subtile»). Les moments de classe où la personne doit nier ou contredire sont relevés comme éprouvants. Argumenter fait donc partie d'un registre difficile dans la mesure où il faut non seulement contredire mais aussi exprimer son opinion. Dans les cultures où l'harmonie est une valeur forte, en particulier dans les cultures asiatiques, les oppositions d'opinion sont vécues comme des ruptures de la relation. «Je ne peux pas dire le contraire de la personne qui vient de parler. C'est impossible pour moi!» Enfin, est évoquée la charge émotionnelle forte devant l'impossibilité de trouver les mots véhiculant l'émotion que l'on veut témoigner. Exprimer sa gratitude, son désaccord, ses condoléances : autant de situations dans lesquelles les personnes ont souffert de ne pas connaitre les codes langagiers permettant de communiquer leurs états internes. Ces difficultés sont liées à l'insuffisance des niveaux lexical et pragmatique. Mais elles entrent directement en jeu dans la qualité des relations avec autrui. Plus grave, le sentiment de perte de cohérence entre le ressenti profond que l'on souhaite exprimer et ce qu'on exprime réellement à l'autre. «Je voulais lui exprimer que $\mathrm{j}$ 'avais de la peine et de la compassion pour elle, mais je ne connaissais ni les mots ni les gestes.» Cette faille entre pensée intime et langage provoque chagrin, déception et sentiment de solitude.

\section{Conclusion}

Ces travaux soulignent l'importance de la prise en compte des émotions dans les activités orales en FLE ou dans des situations d'échanges exolingues. Aider à les expliciter, apprendre à s'en distancier par la prise de conscience des mécanismes «incorporés», donner des outils pour 
analyser l'échange semblent aussi importants et complémentaires que de donner des outils linguistiques. La découverte des composantes pragmatiques de l'échange, associée à la compréhension des choix culturels en œuvre dans les interactions, est fondamentale. Les difficultés soulevées sont valables pour toute entrée dans une communauté nouvelle où les rituels relationnels diffèrent des siens. La prise de conscience de ses fonctionnements intériorisés est favorisée par la décentration que représente l'expatriation. Cela favorise la saisie de nouveaux éléments de sens et le questionnement d'un mode communicationnel incorporé et inconscient. Émergent de nouvelles compétences favorisant la médiation : savoir écouter son ressenti en se distançant des émotions négatives, exprimer ses émotions sous de nouvelles formes (pouvant passer du verbal au non verbal), écouter et percevoir celles d'autrui qui échappaient à la perception immédiate, suspendre le jugement spontané pour accueillir de nouvelles modalités. Les personnes interrogées affirment qu'au fur et à mesure que ces clés de compréhension leur apparaissent, elles développent qualité d'écoute et stabilité sereine dans la relation.

\section{RÉFÉRENCES BIBLIOGRAPHIQUES}

Alber, J.-L. \& Py, B. (1985). Interlangue et conversation exolingue de la communication interculturelle : interparole, coopération et conversation. Cahier du département des langues et des sciences du langage, 1,30-47.

Audibert, N., Aubergé, V. \& Rilliard, A. (2005). The Prosodic Dimensions of Emotion in Speech. The Relative Weights of Parameters. $9^{\mathrm{e}}$ European Conference on Speech Communication and Technology, 525-528.

Austin, J. (2005). Quand dire c'est faire. Paris : Le Seuil.

Auzanneau, M. (2007). La mise en æuvre des langues dans l'interaction. Paris : L'Harmattan.

BÄNZIGER, T. \& SCHERER, K. (2005). The Role of Intonation in Emotional Expressions. Speech Communication, 46, 252-267.

Damasio, A. (2010). L'autre moi-même. Les nouvelles cartes du cerveau, de la conscience et des émotions. Paris : Odile Jacob.

De Pembroke, E. (1998). L'hétérogénéité des représentations du temps dans un cours de langue. Travaux de didactique, 39, 57-66.

Fayol, M. (1997). Des idées au texte. Psychologie cognitive de la production verbale orale et écrite. Paris : PUF.

GaOnaCh, D. (1987). Théories d'apprentissage et acquisition d'une langue étrangère. Paris : Hatier. 
Goffman, E. (1967). Les rites d'interaction. Paris : Minuit.

Gremmo, M.-J. \& Holec, H. (1990). La compréhension orale : un processus et un comportement. Le Français dans le monde. Recherches et applications. Paris : Hachette.

GuMPERz, J. (1989). Sociolinguistique interactionnelle. Une approche interprétative. Paris : L'Harmattan.

Kerbrat-Orecchioni, C. (2008). Les actes de langage dans le discours. Paris : A. Colin.

Levelt, W. (1989). Speaking, from intention to articulation. Cambridge : M.I.T. Press.

Miles, M. \& Huberman, M. (2003). Analyse des données qualitatives. Bruxelles : De Boeck.

Mondillon, L. \& Tcherkassof, A. (2009). La communication émotionnelle : quand les expressions faciales s'en mêlent... Revue électronique de psychologie sociale, 4, 25-31.

Piaget, J. (1974). La prise de conscience. Paris : PUF.

PicARD, D. (1992). Les mécanismes psycho-sociologiques du processus de communication. Cahiers français, 258, 19-25.

Porcher, L. \& Abdallah Pretceille, M. (1998). Éthique de la diversité et éducation. Paris : PUF.

Porquier, R. (1984). Communication exolingue et apprentissage des langues. Encrages, Acquisition d'une langue étrangère III, 17-47.

SEARle, J. (1972). Les actes de langage. Paris : Hermann.

Trévise, A. (1996). Acquisition et interaction en langue étrangère. Activité et représentations métalinguistiques dans les acquisitions des langues. AILE, 8, 5-39.

VAREla, F. (1993). L'inscription corporelle de l'esprit. Paris : Le Seuil.

Vermersch, P. (2008). L'entretien d'explicitation. Paris : ESF. 